\title{
Examination of Intrauterine Inflammation and Immature Platelet Fraction at Birth in Extremely Low Birth Weight Infants
}

\author{
Yuko Sakurai $^{1,2, *}$, Motoichiro Sakurai ${ }^{1}$ \\ ${ }^{1}$ Division of Neonatology, Showa University Koto Toyosu Hospital, Tokyo, Japan \\ ${ }^{2}$ Division of Neonatology, Japanese Red Cross Medical Center, Tokyo, Japan
}

Email address:

nunu0421@med.showa-uac.jp (Y. Sakurai), kii0124@med.showa-u.ac.jp (M. Sakurai)

*Corresponding author

\section{To cite this article:}

Yuko Sakurai, Motoichiro Sakurai. Examination of Intrauterine Inflammation and Immature Platelet Fraction at Birth in Extremely Low Birth Weight Infants. American Journal of Pediatrics. Vol. 6, No. 3, 2020, pp. 334-340. doi: 10.11648/j.ajp.20200603.37

Received: July 27, 2020; Accepted: August 10, 2020; Published: August 18, 2020

\begin{abstract}
IL-6 is thought to be involved in the prognosis of extremely low birth weight infants, in whom it leads to newborn organopathy. IL-6 also influences various processes in vivo, including thrombocytopoiesis. In the present study, we investigated whether the influence of IL-6 in the fetal period could be evaluated using the actual number of infant platelets, which is an index of thrombocytopoiesis. We retrospectively examined the perinatal information of extremely low birth weight infants, IL-6, the number of platelets (Plt), and the number of immature platelets fraction (IPF). The study population included 81 infants. The mean gestational age of the infants was $26.1 \pm 2.5$ weeks and the mean birth weight was $729 \pm 161 \mathrm{~g}$. The immature platelet count (Plt $\times$ IPF) was positively correlated with the IgM level $(\mathrm{R}=0.45, \mathrm{p}<0.01)$ and the membrane rupture time $(\mathrm{R}=0.095, \mathrm{p}<0.01)$. In addition, the Plt $\times$ IPF in the group in which the cord blood IL-6 level was $\geq 11 \mathrm{pg} / \mathrm{mL}$ was significantly higher than that in the group in which the cord blood IL- 6 was $<11 \mathrm{pg} / \mathrm{mL}(\mathrm{p}<0.01)$. The Plt $\times$ IPF value at birth was associated with the IL-6 level in extremely low birth weight infants, which suggests the possibility that it may be used to evaluate fetal inflammation.
\end{abstract}

Keywords: Extra-low Birth Weight Infants, Platelet Count, Immature Platelet Fraction, Chorioamnionitis,

Fetal Inflammatory Response Syndrome (FIRS)

\section{Introduction}

Recently, the survival rate of extremely low birth weight infants has improved, and now efforts are being made to improve the prognosis of neurological development. A number of factors have a major influence on the prognosis of neurological development, including short-term complications, including intraventricular hemorrhage, necrotizing enterocolitis, bronchopulmonary dysplasia, retinopathy of prematurity and periventricular leukomalacia. Regarding these complications, it is reported that the more premature the infant, the stronger the relationship with chorioamnionitis or intrauterine pathogenic microbe infection. Furthermore, in addition to causing premature rupture of the membranes or premature birth, intrauterine inflammation is associated with a younger gestational age and reduced birth weight, indicating a relationship with the short-term prognosis [1].

When inflammation occurs in utero, the levels of various cytokines, including IL-6, increase within the mother's body. In a previous study, the analysis of the blood of mothers who delivered a premature infants based on the presence or absence of histological chorioamnionitis, revealed that the median amniotic fluid IL-6 level in mothers with chorioamnionitis was higher than that in mothers without chorioamnionitis [2]. Furthermore, it is reported that inflammatory cytokines affect the fetus, resulting in hypercytokinemia in the fetus [3]. The breakup of the intrauterine environment leads to the exposure of the fetus to external viruses or inflammation. 
In cord blood, cytokines are mainly produced by monocytes and macrophages. Cytokines are also produced - to a lesser extent—by T lymphocytes, B lymphocytes and neutrophilic cells. Proinflammatory cytokines are involved in the mechanism leading to the damage of the lung and the brain that accompanies inflammation in premature infants, and respiratory distress syndrome and chronic lung disease in newborns are said to be related to the activity levels of cytokines such as IL-6. T-cell activation and cytokine production within the uterus are related to brain lesions in extremely premature newborns. Dembinski et al. [4] reported that the incidence of brain lesions in extremely premature newborns was significantly associated with the IL-6 activity within 24 hours after birth. In other words, the proinflammatory cytokine level increases through the mother, and the response of the fetus itself causes impairment of the organ function or inhibition of the normal development of the fetus, which manifests as various diseases, including chronic lung disease, after birth [1]. This is recognized as fetal inflammatory response syndrome (FIRS) and an increase in the levels of cytokines, such as IL-6, is thought to lead to organopathy [5].

Cytokines that increase with fetal inflammation are also involved in thrombocytopoiesis. Many substances have been reported to be associated with thrombocytopoiesis, including IL-6. We hypothesized that the platelet count and immature platelet fraction (IPF) might predict the level of inflammation. However, it is a fact that thrombocytopoiesis is affected by many factors, including the gestational age at birth, birth weight and SD score [8]. Therefore, in an individual comparison of the platelet count or IPF, it is necessary to consider multiple factors. We therefore focused on the real number of immature platelets, which is the product of the platelet count and IPF, as a new index, and examined whether it was influenced by gestational age, birth weight or SD score. We also evaluated the relationship with intrauterine infection.

\section{Materials and Methods}

The study population included extremely low birth weight infants who were admitted to the Japanese Red Cross Medical Center NICU between January 2013 and December 2017. The inclusion criteria were hospitalization within 24 hours after birth and the absence of chromosome aberrations or anomalies.

Based on the patients' medical records, we retrospectively examined the stage of premature rupture of membranes, the time to delivery, gestational age, birth weight, Apgar score, placental pathological findings, cord blood IgM and IL-6, platelet counts and IPF within 24 hours after birth. The platelet count and IPF value were measured using an XE-2100 multiparameter automated hematology analyzer (Sysmex Corp.).

We investigated whether there was a correlation between the product of the platelet count and IPF value (Plt $\times$ IPF) and the examined factors. Moreover, using a cut-off value of IL-6 of 11 $\mathrm{pg} / \mathrm{mL}$, which is a criterion for FIRS, we examined Plt $\times$ IPF.

The Mann-Whitney U test, Fisher's direct probability and Spearman's rank-order correlation coefficient matrix were used as statistical analyses. In all analyses, two-tailed $\mathrm{p}$ values of $<0.05$ were considered to be statistically significant. All statistical analyses were performed using BellCurve for Excel (v. 3.20; Social Survey Research Information Co. Ltd.).

This study is approved by Japanese Red Cross Medical Center organization screening committee.

\section{Results}

The characteristics of the 81 infants examinedare shown in Table 1. Forty-one of the infants $(50 \%)$ were male, the mean gestational age was $26.1 \pm 2.5$ weeks, and the mean birth weight was $729 \pm 161 \mathrm{~g}$. The mean SD score was $-0.97 \pm 1.52$, the mean IPF value was $5.0 \pm 2.3 \%$ and the mean Plt $\times$ IPF value was $98.3 \pm 50.8 \times 104 / \mu \mathrm{L}$.

Table 1. Overall characteristics that was the subjects of this study.

\begin{tabular}{ll}
\hline & Subjects $(\mathbf{N}=\mathbf{8 1})$ \\
\hline Male infant & $41(50 \%)$ \\
Gestational age & $26.1 \pm 2.5$ \\
Birth weight & $729 \pm 161$ \\
SD score & $-0.97 \pm 1.52$ \\
Apgar score at 1 minute & $4 \pm 2$ \\
Apgar score at 5 minutes & $7 \pm 1$ \\
Primiparity & $49(60 \%)$ \\
Mode of delivery vaginal birth & $24(30 \%)$ \\
Cesarean section & $57(70 \%)$ \\
platelet count $\left(\times 10^{4} / \mu \mathrm{L}\right)$ & $21.4 \pm 9.6$ \\
IPF $(\%)$ & $5.0 \pm 2.3$ \\
platelet count $\times \operatorname{IPF}\left(\times 10^{2} / \mu \mathrm{L}\right)$ & $98.3 \pm 50.8$ \\
IgM (mg/dL) & Median $6(2-227)$ \\
Cord blood IL-6 $(\mathrm{ng} / \mathrm{mL})$ & Median $17.0(1.0-10810.0)$ \\
CAM stage 1 & $10(12 \%)$ \\
CAM stage 2 & $7(8 \%)$ \\
CAM stage 3 & $26(32 \%)$ \\
\hline
\end{tabular}

IPF; Immature platelet fraction, CAM; Chorioamnionitis. 
The analysis of the correlations between the Plt $\times$ IPF value, and the gestational age, birth weight, SD score, cord blood IgM value, time (membrane rupture time) from membrane rupture to delivery, and the ante partum mother CRP value, are

$$
\text { Gestational age (weeks) }
$$

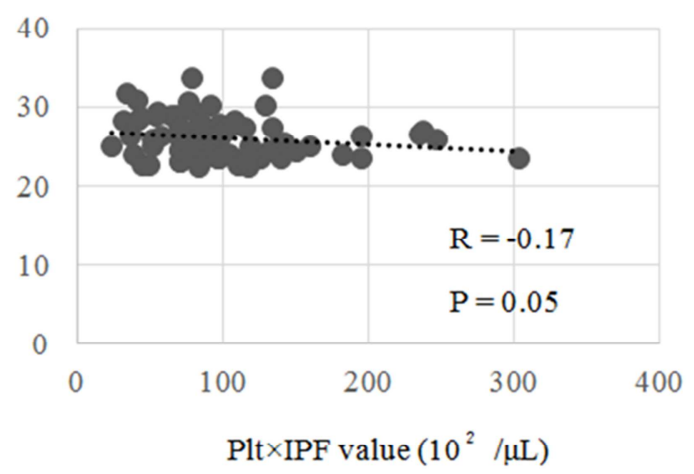

(a) Plt $\times$ IPF and gestational age

\section{SD score}

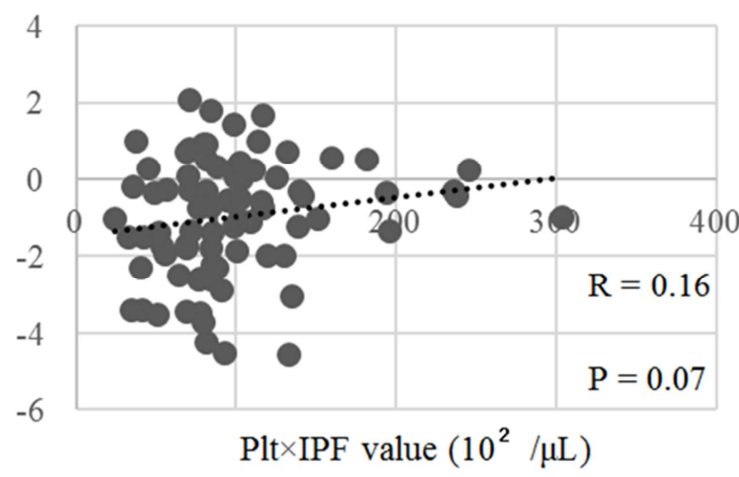

(c) Plt $\times$ IPF and SD score

Membrane rupture time (time)

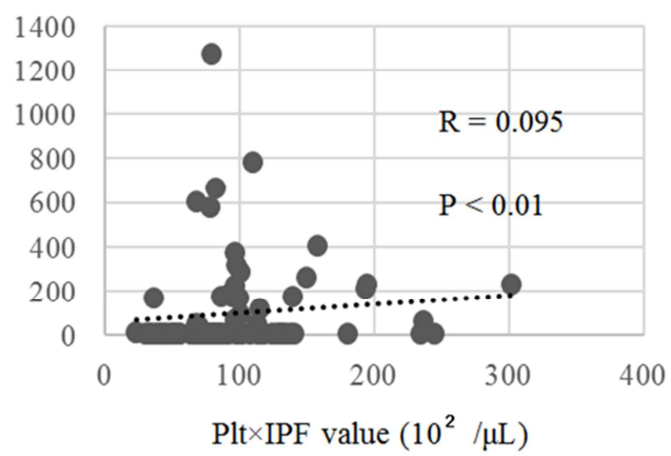

(e) Plt $\times$ IPF and membrane rupture time shown in Figure 1. Significant correlations were recognized between Plt $\times$ IPF and $\operatorname{IgM}(\mathrm{R}=0.45, \mathrm{p}<0.01)$ and the membrane rupture time $(\mathrm{R}=0.095, \mathrm{p}<0.01)$.

Birth weight (g)

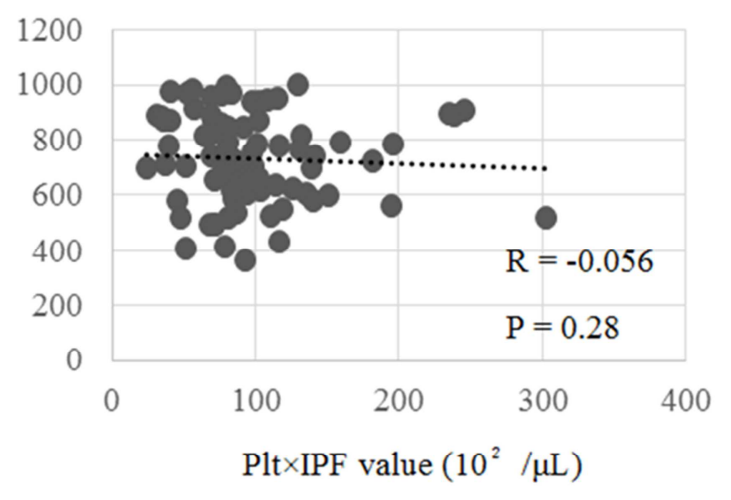

(b) Plt $\times$ IPF and birth weight

$\operatorname{IgM}(\mathrm{mg} / \mathrm{dL})$

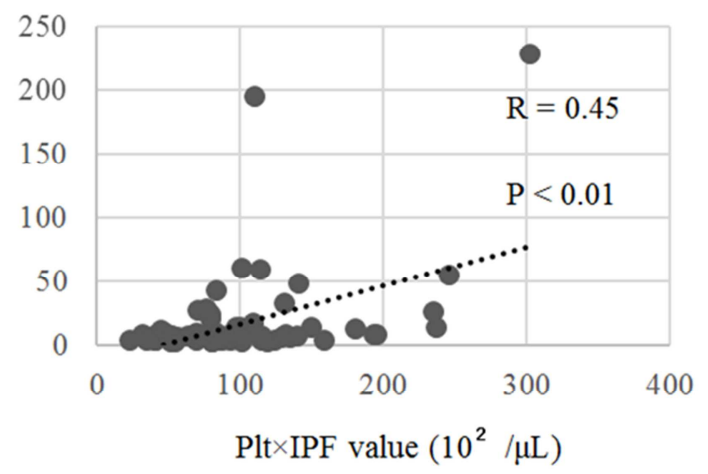

(d) Plt $\times$ IPF and IgM

CRP (mg/dL)

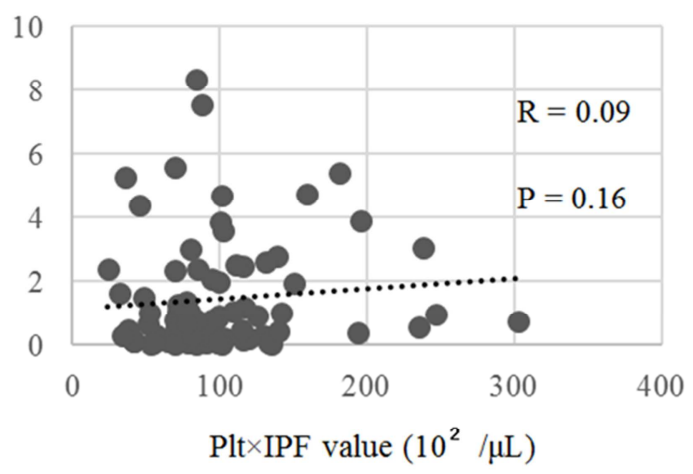

(f) Plt $\times$ IPF and maternal CRP

Figure 1. Correlation between Plt $\times I P F$ and each item Correlations are observed between Plt $\times I P F$ and gestational age, birth weight, SD score, IgM, membrane rupture time and maternal CRP.

Regarding the relationship between cord blood IL-6 and Plt $\times$ IPF, the Plt $\times$ IPF value in the IL- $6 \geq 11 \mathrm{pg} / \mathrm{mL}$ group was significantly higher than that in the IL-6 $<11 \mathrm{pg} / \mathrm{mL}$ group $(\mathrm{p}<0.01)$ (Figure 2, Table 2). 
Table 2. Characteristics in patients with IL-6 levels of $\geq 11 \mathrm{pg} / \mathrm{mL}$ and $<11 \mathrm{pg} / \mathrm{mL}$.

\begin{tabular}{llll}
\hline & IL-6 levels of $\geq \mathbf{1 1} \mathbf{~ p g} / \mathbf{m L}(\mathbf{N}=\mathbf{3 5})$ & IL-6 levels of $<\mathbf{1 1} \mathbf{~ p g} / \mathbf{m L}$ (N=46) & p value \\
\hline Male infant & $15(42 \%)$ & $26(56 \%)$ & 0.26 \\
Gestational age & $27.1 \pm 2.3$ & $25.4 \pm 2.4$ & $<0.01$ \\
Birth weight & $749 \pm 171$ & $714 \pm 154$ & 0.38 \\
SD score & $-1.54 \pm 1.45$ & $-0.54 \pm 1.43$ & $<0.01$ \\
platelet count $\left(\times 10^{4} / \mu \mathrm{L}\right)$ & $16.9 \pm 7.3$ & $24.8 \pm 9.7$ & $<0.01$ \\
IPF $(\%)$ & $5.0 \pm 2.0$ & $5.0 \pm 2.6$ & 0.57 \\
platelet count $\times$ IPF $\left(\times 10^{2} / \mu \mathrm{L}\right)$ & $77.3 \pm 26.2$ & $114.3 \pm 58.9$ & $<0.01$ \\
IgM $(\mathrm{mg} / \mathrm{dL})$ & Median $6(2-58)$ & Median $8(2-227)$ & 0.04 \\
\hline
\end{tabular}

IPF; Immature platelet fraction.

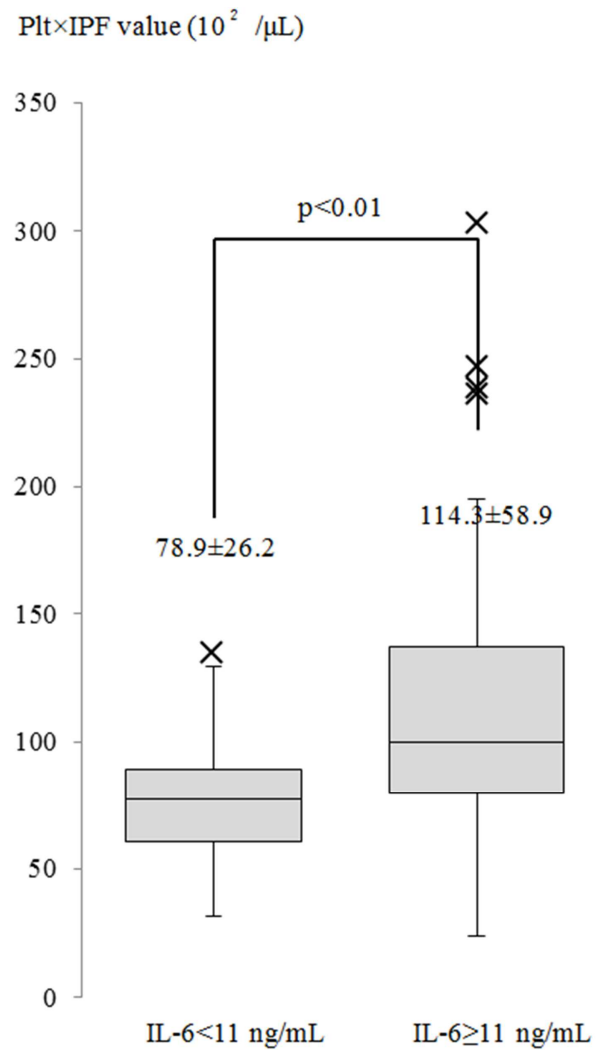

Figure 2. Plt $\times I P F$ and cord blood IL-6 Plt $\times I P F$ values in patients with IL-6 levels of $\geq 11 \mathrm{pg} / \mathrm{mL}$ and $<11 \mathrm{pg} / \mathrm{mL}$.

\section{Discussion}

There was no correlation between Plt $\times$ IPF and gestational age, birth weight or SD score; however, positive correlations were recognized between Plt $\times$ IPF and IgM as well as the membrane rupture time. When cord blood IL-6, which is a criterion of FIRS (along with maternal intrauterine inflammation) was compared using a cut-off value of 11 $\mathrm{pg} / \mathrm{mL}[9]$, the Plt $\times$ IPF value was significantly higher in the IL- $6 \geq 11 \mathrm{pg} / \mathrm{mL}$ group.

It has been pointed out that chorioamnionitis is highly associated with spontaneous preterm birth and abortion in early to intermediary pregnancy [1]. When a microbe invades the amniotic cavity, a strong local inflammation response is induced, and the concentrations of proinflammatory cytokines, such as IL-6 remarkably increase $[1,10,11]$. It has been shown that proinflammatory cytokines play an important role in the pathogenesis of tissue lesions and organ dysfunction under circumstances such as neonatal infection, hypoxia and ischemia. Among these cytokines, IL-6 is a core cytokine with multifaceted effects in the cytokine cascade $[12,13]$.

Generally, the CRP value is used as an index of inflammation. However, the maternal CRP value is not associated with chorioamnionitis, and it is considered to have low prognostic value in cases of intrauterine inflammation [14]. The present study revealed relationships between Plt $\times$ IPF and the maternal membrane rupture time, IgM value of the infant and cord blood IL-6 value, rather than the maternal CRP value. Cytokines are the main mediator of acute phase reactions, and IL- 6 is considered to be very important as an index of inflammatory influence on the fetus [15-18], leading to the concept of FIRS [19]. The diagnostic criteria for FIRS, reported Gomez et al. [9], include an intranatal cord blood IL-6 value of $\geq 11 \mathrm{pg} / \mathrm{mL}$. Increased cytokine levels cause result in decreased biophysical activities, which manifest as a tendency to somnolence or decreased breathing exercise, as effects of the central nervous system. These are considered to be "clinical symptoms of the fetus" in FIRS, while premature birth and PPROM are thought to be maternal symptoms [9]. Furthermore, FIRS is related to the risk of BPD, PVL and NEC of premature infants [1, 2, 20].

Recently, in cases of acute chorioamnionitis with or without microorganisms [1], danger signals of non-microbial origin have been thought to be a main factor in sterile intra-amniotic inflammation [21]. Thus, intra-amniotic inflammation is diagnosed based on an amniotic fluid IL- 6 value of $\geq 2.6 \mathrm{ng} / \mathrm{ml}$ [21]. However, we refer to the pathological diagnosis of chorioamnionitis after birth because of the test is associated with risks in the mother and infant, and is less precise than a pathological examination.

Thus, IgM is an important blood test value in premature infants. Since IgM does not have placental permeability, the IgM value of the cord blood is reported to be approximately one-tenth of the adult level [19]. In addition, the fetus has the ability to produce IgM. IL-6 elevation stimulates the B cells of the fetus, which promotes antibody production and an increase in IgM. The measurement of the newborn blood IgM value is therefore important for the diagnosis of infectious disease in utero [19]. In CAM, in 
which the incidence rates of intrauterine infection and inflammation are high, the serum IgM value has been reported to be increased and to be predominately related to BPD [22, 23], and the serum IgM value is reported to be useful for predicting the prognosis of newborns. However, similarly to other in vivo-produced substances, the production of IgM takes time, and it is therefore impossible to judge whether the intranatal $\operatorname{IgM}$ value is the actual maximum IgM value based on a single measurement at birth. Consequently, the ability to predict the neonatal prognosis based on IgM alone is limited.

Regarding the time required for thrombocytopoiesis in humans, it is reported that it takes approximately five days for megakaryocytes to complete polyploidization of platelets, mature and release platelets [24]. In a murine study, the peak of thrombocytopoiesis for the production of IL-6 was reported to occur at around 10 days [7]. In other words, we need to combine the results of tests that are measurable at birth to assess the inflammation exposure of the fetus or predict the prognosis of the newborn in greater detail. We connected these processes of intra-amniotic inflammation to the fetal immunologic response as well as the hemopoiesis mechanism, as illustrated in Figure 3. In an infant in whom IL-6 was increased due to intra-amniotic inflammation, which led to premature birth, we hypothesized that we could indirectly evaluate the proinflammatory cytokine levels at birth by IPF, and that this might be factor that predicts whether increased IL-6 will cause PVL or BPD.

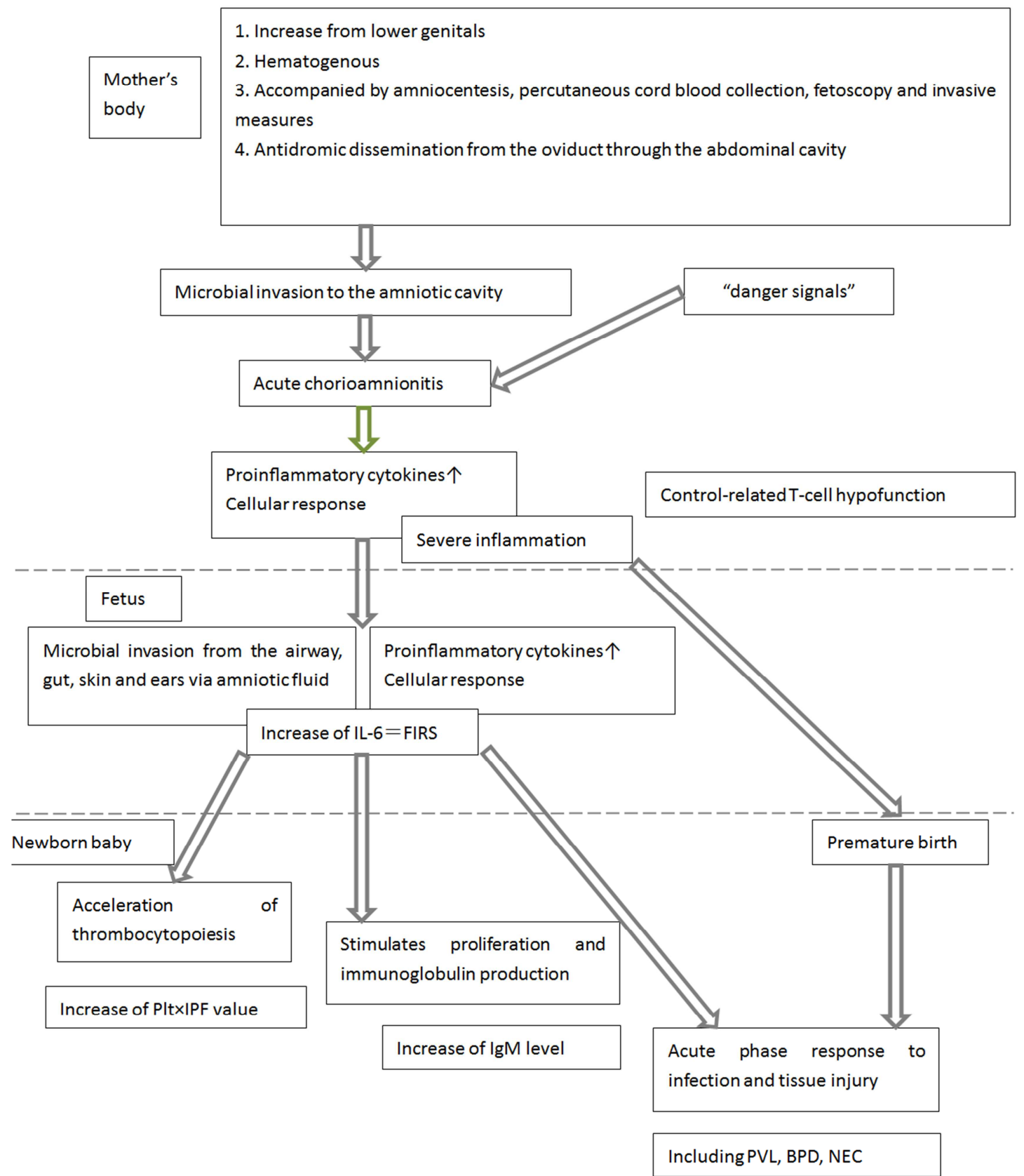

Figure 3. The influence of acute chorioamnionitis from the fetal period to birth Maternal inflammation spreads to the fetus, leading to FIRS, in which IL-6 increases. This shows the relationship between the increase in IL-6 in the fetus and intranatal IgM and an immature platelet count (Plt $\times I P F)$. 
Thrombocytopoiesis is related to various factors including cytokines. IL-6 also interacts with various factors and regulates the growth of progenitor cells in the late phase of hematopoiesis [6, 7]. Meanwhile, we previously reported that IPF, an index of thrombocytopoiesis, is affected both the platelet count and the condition of the infant, including the gestational age, birth weight and SD score [8].

Thus, in the present study, we hypothesized that Plt $\times$ IPF could be used as a new index. There is a negative correlation between the platelet count and IPF. We considered this because the Plt $\times$ IPF value showed the actual IPF value and more subtly represented the influence of IL-6 on megakaryocytic maturity and production. Actually, there was no correlation between the Plt $\times$ IPF value and gestational age, birth weight or SD score, whereas relationships were found with IgM, membrane rupture time and IL-6, which are indices that suggest intrauterine inflammation. These results suggest that Plt $\times$ IPF value may be a useful index that indirectly indicates intrauterine inflammation.

The present study was associated with some limitations. The targeted infants were from a single institution and the study population was relatively small. Furthermore, with regard to intrauterine inflammation, only the pathological diagnosis of CAM was used, and we did not investigate whether the patients had sterile or bacterial intrauterine inflammation, such as by performing tests to investigate the presence or absence of a primary causative organism. Nevertheless, since performing a detailed analysis of the inflammatory cytokines in a newborn baby requires large blood samples, it is thought that this value, which can be evaluated by a simple test that is necessary to perform on admission, is a useful index.

Future studies are expected to investigate the detailed causes of preterm birth and the relationship with other factors. We expect that the Plt $\times$ IPF value will be used as a new index to predict the prognosis of premature infants.

\section{Conclusion}

A positive correlation was found between the product of platelet count and IPF at birth in extremely low birth weight infants, neonatal IgM, and the maternal membrane rupture time at birth. In addition, based on the classification by cord blood IL-6, the Plt $\times$ IPF value was found to be significantly high in the group that met the criteria of FIRS. The results suggest that the Plt $\times$ IPF value might be useful for predicting the prognosis of preterm infants.

\section{Statements}

\section{Statement of Ethics}

This study is approved by Japanese Red Cross Medical Center organization screening committee. And the subjects (or their parents or guardians) have given their written informed consent.

\section{Conflict of Interest Statement}

All the authors do not have any possible conflicts of interest.

\section{Funding Sources}

None.

\section{References}

[1] Kim CJ, Romero R, Chaemsaithong P, Chaiyasit N, Yoon BH, Kim YM. Acute chorioamnionitis and funisitis: definition, pathologic features, and clinical significance. Am J Obstet Gynecol 2015 Oct; 213 (4 Suppl): S29-52.

[2] Romero R, Miranda J, Chaiworapongsa T, Korzeniewski SJ, Chaemsaithong P, Gotsch F, et al. Prevalence and clinical significance of sterile intra-amniotic inflammation in patients with preterm labor and intact membranes. Am J Reprod Immunol 2014 Nov; 72 (5): 458-74.

[3] Kim CJ, Yoon BH, Romero R, Moon JB, Kim M, Park SS, et al. Umbilical arteritis and phlebitis mark different stages of the fetal inflammatory response. Am J Obstet Gynecol 2001 Aug; 185 (2): 496-500.

[4] Dembinski J, Martini R, Dembinski J, Martini R, Behrendt D, Bartmann P. Modification of cord blood IL-6 production with IgM enriched human immunoglobulin in term and preterm infants. Cytokine 2004 Apr 7; 26 (1): 25-9.

[5] Pacora P, Chaiworapongsa T, Maymon E, Kim YM, Gomez R, Yoon $\mathrm{BH}$, et al. Funisitis and chorionic vasculitis: the histological counterpart of the fetal inflammatory response syndrome. J Matern Fetal Neonatal Med 2002 Jan; 11 (1): 18-25.

[6] Deutsch VR, Tomer A. Advances in megakaryocytopoiesis and thrombopoiesis: from bench to bedside. Br J Haematol 2013 Jun; 161 (6): 778-93.

[7] Wu D, Xie J, Wang X, Zou B, Yu Y, Jing T, et al. Micro-concentration lipopolysaccharide as a novel stimulator of megakaryocytopoiesis that synergizes with IL- 6 for platelet production. Sci Rep 2015 Sep 2; 5: 13748.

[8] Yuko S, Takeda T, Hirota A, Hisaeda Y, Amakata S, Nakao A, et al. Examination of the percentage of immature platelet fraction in term and preterm infants at birth. J Clin Neonatol 2013 Oct; 2 (4): 173-8.

[9] Gomez R, Romero R, Ghezzi F, Yoon BH, Mazor M, Berry SM The fetal inflammatory response syndrome. Am J Obstet Gynecol 1998 Jul; 179 (1): 194-202.

[10] Romero R, Avila C, Santhanam U, Sehgal P. B. Amniotic fluid interleukin 6 in preterm labor. Association with infection. J Clin Invest 1990 May; 85 (5): 1392-400.

[11] Sorokin Y, Romero R, Mele L, Iams JD, Peaceman AM, Leveno KJ, et al. Umbilical cord serum interleukin-6, C-reactive protein, and myeloperoxidase concentrations at birth and association with neonatal morbidities and long-term neurodevelopmental outcomes. Am J Perinatol 2014 Sep; 31 (8): 717-26. 
[12] Leon LR. Invited review: cytokine regulation of fever: studies using gene knockout mice. J Appl Physiol (1985) 2002 Jun; 92 (6): 2648-55.

[13] Jones TH, Kennedy RL. Cytokines and hypothalamic-pituitary function. Cytokine 1993 Nov; 5 (6): 531-8.

[14] Musilova I, Kacerovsky M, Stepan M, Bestvina T, Pliskova L, Zednikova B, et al. Maternal serum C-reactive protein concentration and intra-amniotic inflammation in women with preterm prelabor rupture of membranes. PLoS One 2017 Aug 16; 12 (8): e0182731.

[15] Dudley DJ. Pre-term labor: an intra-uterine inflammatory response syndrome? J Reprod Immunol 1997 Nov 30; 36 (1-2): 93-109.

[16] Goldenberg RL, Culhane JF, Iams JD, Romero R. Epidemiology and causes of preterm birth. Lancet 2008 Jan 5; 371 (9606): 75-84.

[17] Iams JD, Romero R, Culhane JF, Goldenberg RL. Primary, secondary, and tertiary interventions to reduce the morbidity and mortality of preterm birth. Lancet 2008 Jan 12; 371 (9607): 164-75.

[18] Waters TP, Mercer BM. The management of preterm premature rupture of the membranes near the limit of fetal viability. Am J Obstet Gynecol 2009 Sep; 201 (3): 230-40.
[19] Namba F, Kitajima H, Tabata A, Nakayama M, Suehara N, Matsunami K, et al. Anti-annexin A2 IgM antibody in preterm infants: its association with chorioamnionitis. Pediatr Res 2006 Dec; 60 (6): 699-704.

[20] Tchirikov M, Schlabritz-Loutsevitch N, Maher J, Buchmann J, Naberezhnev Y, Winarno AS, et al. Mid-trimester preterm premature rupture of membranes (PPROM): etiology, diagnosis, classification, international recommendations of treatment options and outcome. J Perinat Med 2018 Jul 26; 46 (5): $465-488$.

[21] Romero R, Miranda J, Chaiworapongsa T, Chaemsaithong P, Gotsch F, Dong Z, et al. Sterile intra-amniotic inflammation in asymptomatic patients with a sonographic short cervix: prevalence and clinical significance. J Matern Fetal Neonatal Med 2015 Jul; 28 (11): 1343-1359.

[22] Fujimura M, Takeuchi T, Ando M, Funato M, Shimada S, Tsujimoto A, et al. Elevated immunoglobulin $\mathrm{M}$ levels in low birth-weight neonates with chronic respiratory insufficiency. Early Hum Dev 1983 Dec; 9 (1): 27-32.

[23] Fujimura M, Takeuchi T, Kitajima H, Nakayama M. Chorioamnionitis and serum IgM in Wilson-Mikity syndrome. Arch Dis Child 1989 Oct; 64 (10 Spec No): 1379-83.

[24] Machlus KR, Italiano JE Jr. The incredible journey: From megakaryocyte development to platelet formation. J Cell Biol 2013 Jun 10; 201 (6): 785-96. 